\title{
New and refined bounds for expected maxima of fractional Brownian motion
}

\author{
Konstantin Borovkov ${ }^{1}$, Yuliya Mishura ${ }^{2}$, \\ Alexander Novikov ${ }^{3}$ and Mikhail Zhitlukhin ${ }^{4}$
}

\begin{abstract}
For the fractional Brownian motion $B^{H}$ with the Hurst parameter value $H$ in $(0,1 / 2)$, we derive new upper and lower bounds for the difference between the expectations of the maximum of $B^{H}$ over $[0,1]$ and the maximum of $B^{H}$ over the discrete set of values $i n^{-1}, i=1, \ldots, n$. We use these results to improve our earlier upper bounds for the expectation of the maximum of $B^{H}$ over $[0,1]$ and derive new upper bounds for Pickands' constant.
\end{abstract}

Key words and phrases: fractional Brownian motion, convergence rate, discrete time approximation, Pickands' constant.

AMS Subject Classification: 60G22, 60G15, 60E15.

\section{Introduction}

Let $B^{H}=\left(B_{t}^{H}\right)_{t \geq 0}$ be a fractional Brownian motion (fBm) process with Hurst parameter $H \in(0,1)$, i.e. a zero-mean continuous Gaussian process with the covariance function $\mathbf{E} B_{s}^{H} B_{t}^{H}=\frac{1}{2}\left(s^{2 H}+t^{2 H}-|s-t|^{2 H}\right), s, t \geq 0$. Equivalently, the last condition can be stated as $B_{0}^{H}=0$ and

$$
\mathbf{E}\left(B_{s}^{H}-B_{t}^{H}\right)^{2}=|s-t|^{2 H}, \quad s, t \geq 0 .
$$

Recall that the Hurst parameter $H$ characterizes the type of the dependence of the increments of the fBm. For $H \in\left(0, \frac{1}{2}\right)$ and $H \in\left(\frac{1}{2}, 1\right)$, the increments of $B^{H}$ are respectively negatively and positively correlated, whereas the process $B^{1 / 2}$ is the standard Brownian motion which has independent increments. The fBm

\footnotetext{
${ }^{1}$ School of Mathematics and Statistics, The University of Melbourne, Parkville 3010, Australia; e-mail: borovkov@unimelb.edu.au.

${ }^{2}$ Mechanics and Mathematics Faculty, Taras Shevchenko National University of Kyiv, Volodymyrska str. 64, 01601 Kyiv, Ukraine; email: myus@univ.kiev.ua.

${ }^{3}$ School of Mathematical and Physical Sciences, University of Technology Sydney, PO Box 123, Broadway, Sydney, NSW 2007, Australia; email: Alex.Novikov@uts.edu.au.

${ }^{4}$ Steklov Mathematical Institute of Russian Academy of Sciences, Gubkina str. 8, 119991, Moscow, Russia; email: mikhailzh@mi.ras.ru.
} 
processes are important construction blocks in various application areas, the ones with $H>\frac{1}{2}$ being of interest as their increments exhibit long-range dependence, while it was shown recently that fBm's with $H<\frac{1}{2}$ can be well fitted to real life telecommunications, financial markets with stochastic volatility and other financial data (see, e.g., [2, 3]). For detailed exposition of the theory of fBm processes, we refer the reader to [4, 8, 9] and references therein.

Computing the value of the expected maximum

$$
M^{H}:=\mathbf{E} \max _{0 \leq t \leq 1} B_{t}^{H}
$$

is an important question arising in a number of applied problems, such as finding the likely magnitude of the strongest earthquake to occur this century in a given region or the speed of the strongest wind gust a tall building has to withstand during its lifetime etc. For the standard Brownian motion $B^{1 / 2}$, the exact value of the expected maximum is $\sqrt{\pi / 2}$, whereas for all other $H \in(0,1)$ no closed-form expressions for the expectation are known. In the absence of such results, one standard approach to computing $M^{H}$ is to evaluate instead its approximation

$$
M_{n}^{H}:=\mathbf{E} \max _{1 \leq i \leq n} B_{i / n}^{H}, \quad n \geq 1,
$$

(which can, for instance, be done using simulations) together with the approximation error

$$
\Delta_{n}^{H}:=M^{H}-M_{n}^{H} .
$$

Some bounds for $\Delta_{n}^{H}$ were recently established in [5]. The main result of the present note is an improvement of the following upper bound for $\Delta_{n}^{H}$ obtained in Theorem 3.1 of [5]: for $n \geq 2^{1 / H}$,

$$
\Delta_{n}^{H} \leq \frac{2(\ln n)^{1 / 2}}{n^{H}}\left(1+\frac{4}{n^{H}}+\frac{0.0074}{(\ln n)^{3 / 2}}\right) .
$$

Lower bound for $\Delta_{n}^{H}$ is obtained as well and we study for which $H$ and $n$ upper and lower bounds hold simultaneously. We also obtain a new upper bound for the expected maximum $M^{H}$ itself and some functions of it, which refines previously known results (see e.g. [5, 12]), and use it to derive an improved upper bound for the so-called Pickands' constant, which is the basic constant in the extreme value theory of Gaussian processes.

The paper is organized as follows: Section 2 contains the results, with comments and examples, and Section 3 contains the proofs.

\section{Main results}

From now on, we always assume that $H \in\left(0, \frac{1}{2}\right)$. The next theorem is the main result of the note. As usual, $\lfloor x\rfloor$ and $\lceil x\rceil$ denote the floor and the ceiling of the real number $x$. 
Theorem 1. 1) For any $\alpha>0$ and $n \geq 2^{1 / \alpha} \vee\left(1+\frac{\alpha}{1+\alpha}\right)^{1 /(2 \alpha H)}$ one has

$$
\frac{\Delta_{n}^{H}}{n^{-H}(\ln n)^{1 / 2}} \leq \frac{\left(1-\left\lfloor n^{\alpha}\right\rfloor^{-1}\right)^{H}(1+\alpha)^{1 / 2}}{1-\left\lfloor n^{\alpha}\right\rfloor^{-H}(1+\alpha /(1+\alpha))^{1 / 2}}
$$

2) For any $n \geq 2$ one has

$$
\frac{\Delta_{n}^{H}}{n^{-H}(\ln n)^{1 / 2}} \geq n^{H}\left(\frac{L}{\left(\ln n^{H}\right)^{1 / 2}}-1\right)^{+},
$$

where $L=1 / \sqrt{4 \pi e \ln 2} \approx 0.2$ and $a^{+}=a \vee 0$.

Remark 1. Note that inequality (4) actually holds for all $H \in(0,1)$.

Remark 2. Let us study for which $H$ and $n$ upper and lower bounds (3) and (44) hold simultaneously under assumption that (4) is non-trivial. For non-triviality we need to have $n<\exp \frac{L^{2}}{H}$. In order to have $2^{1 / \alpha} \leq \exp \frac{L^{2}}{H}$ we restrict $\alpha$ to $\alpha \geq \frac{H \ln 2}{L^{2}}$. In order to have $\left(1+\frac{\alpha}{1+\alpha}\right)^{1 /(2 \alpha H)} \leq \exp \left\{\frac{L^{2}}{H}\right\}$, or, what is equivalent,

$$
\left(1+\frac{\alpha}{1+\alpha}\right)^{1 / \alpha} \leq \exp \left\{2 L^{2}\right\}
$$

we note that the function $q(\alpha)=\left(1+\frac{\alpha}{1+\alpha}\right)^{1 / \alpha}$ continuously strictly decreases in $\alpha \in(0, \infty)$ from $e$ to 1 , and taking into account the value of $L$, we get that there is a unique root $\alpha^{*} \approx 7.48704$ of the equation $\left(1+\frac{\alpha}{1+\alpha}\right)^{1 / \alpha}=\exp \left\{2 L^{2}\right\}$ and for $\alpha \geq \alpha^{*}$ we have that (5) holds. Therefore for $\alpha>\alpha^{*}, H<\frac{\alpha^{*} L^{2}}{\ln 2} \approx 0.456$ and $\exp \left\{\frac{L^{2}}{H}\right\}>n>2^{1 / \alpha} \vee\left(1+\frac{\alpha}{1+\alpha}\right)^{1 /(2 \alpha H)}$ we have that lower bound (4) holds and is non-trivial. Moreover, $2^{1 / \alpha}<2^{1 / \alpha^{*}}(\approx 1.097)<\exp \left\{\frac{L^{2}}{H}\right\},\left(1+\frac{\alpha}{1+\alpha}\right)^{1 /(2 \alpha H)}<$ $\left(1+\frac{\alpha^{*}}{1+\alpha^{*}}\right)^{1 /\left(2 \alpha^{*} H\right)}=\exp \left\{\frac{L^{2}}{H}\right\}$, so the interval $\left(2^{1 / \alpha} \vee\left(1+\frac{\alpha}{1+\alpha}\right)^{1 /(2 \alpha H)}, \exp \left\{\frac{L^{2}}{H}\right\}\right)$ is non-empty and for such $n$ upper bound (3) holds. The only question is if this interval contains the integers. If it is not the case we can increase the value of $\alpha$. For example, put $H=0.01, \alpha=16$, then it holds that the interval $\left(2^{1 / \alpha} \vee(1+\right.$ $\left.\left.\frac{\alpha}{1+\alpha}\right)^{1 /(2 \alpha H)}, \exp \left\{\frac{L^{2}}{H}\right\}\right)=(1.044 \vee 7.534,20.085)=(7.534,20.085)$.

Remark 3. Consider the sequence $\alpha=\alpha(m) \rightarrow 0$ slowly enough as $m \rightarrow \infty$ (take, e.g., $\alpha(m)=(\ln \ln m) / \ln m)$. Then for sufficiently large enough $m$ we have that $m \geq 2^{1 / \alpha(m)} \vee\left(1+\frac{\alpha(m)}{1+\alpha(m)}\right)^{1 /(2 \alpha(m) H)}$ therefore for such $m$ the upper bound (3) holds. Returning to standard notation $n$ for the argument, we obtain from the upper bound in (3) that, for any fixed $H \in\left(0, \frac{1}{2}\right)$, one has

$$
\Delta_{n}^{H} \leq n^{-H}(\ln n)^{1 / 2}(1+o(1)), \quad n \rightarrow \infty
$$

which refines (2). 
Remark 4. Recall that, in the case of the standard Brownian motion $\left(H=\frac{1}{2}\right)$, the exact asymptotics of $\Delta_{n}^{1 / 2}$ are well-known:

$$
\Delta_{n}^{1 / 2}=n^{-1 / 2}(\beta+o(1)), \quad n \rightarrow \infty,
$$

where $\beta=-\zeta(1 / 2) / \sqrt{2 \pi}=0.5826 \ldots$ and $\zeta(\cdot)$ is the Riemann zeta function (see [13]). Comparing it with (6), we see that now we have additional logarithmic multiplier.

The next simple assertion enables one to use the upper bound obtained in Theorem 1 to get an upper bound for the approximation rate of the expectation of a function of the maximum of an $\mathrm{fBm}$. Such a result is required, for instance, for bounding convergence rates when approximating Bayesian estimators in irregular statistical experiments (see, e.g., [7, 10]).

Set

$$
\bar{B}_{1}^{H}:=\max _{0 \leq t \leq 1} B_{t}^{H}, \quad \bar{B}_{n, n}^{H}:=\max _{1 \leq i \leq n} B_{i / n}^{H}, \quad \Delta_{n}^{H, f}:=\mathbf{E} f\left(\bar{B}_{1}^{H}\right)-\mathbf{E} f\left(\bar{B}_{n, n}^{H}\right)
$$

and, for a function $f: \mathbb{R} \rightarrow \mathbb{R}$, denote its continuity modulus by

$$
\omega_{\delta, h}(f):=\sup _{0 \leq s<t \leq(s+\delta) \wedge h}|f(s)-f(t)|, \quad h, \delta>0 .
$$

Corollary 1. Let $f \geq 0$ be an arbitrary non-decreasing function on $\mathbb{R}$ such that $f(x)=o\left(\exp \left(\left(x-M^{H}\right)^{2} / 2\right)\right)$ as $x \rightarrow \infty$. Then, for any number $M>M^{H}$,

$$
\Delta_{n}^{H, f} \leq \omega_{\Delta_{n}^{H}, M}(f)+\int_{M}^{\infty} f(x)\left(x-M^{H}\right) \exp \left\{-\left(x-M^{H}\right)^{2} / 2\right\} d x .
$$

To roughly balance the contributions from the two terms in the bound, one may wish to choose $M$ so that $\exp \left\{-\left(M-M^{H}\right)^{2} / 2\right\}$ would be of the same order of magnitude as $\Delta_{n}^{H}$ (as for regular functions $f$ that are mostly of interest in applications are locally Lipschitz, so that $\omega_{\delta, h}(f)$ admits a linear upper bound in $\delta$ ). To that end, one can take $M:=M^{H}+\left(-2 \ln \Delta_{n}^{H}\right)^{1 / 2}+$ const (assuming that $n$ is large enough so that $\left.\Delta_{n}^{H}<1\right)$. We will illustrate that in two special cases where $f$ is the exponential function (this case corresponds to the above-mentioned applications from [7, 10]) and a power function, respectively.

Example 1. Assume that $f(x)=e^{a x}$ with a fixed $a>0$, and that $\Delta_{n}^{H}<1$. Choosing $M:=M^{H}+a+\left|2 \ln \Delta_{n}^{H}\right|^{1 / 2}$ we get

$$
\omega_{\Delta_{n}^{H}, M}(f) \leq e^{a M} \Delta_{n}^{H}=\exp \left\{a M^{H}+a^{2}+a\left|2 \ln \Delta_{n}^{H}\right|^{1 / 2}\right\} \Delta_{n}^{H}
$$

and, setting $y:=x-M^{H}$ and using the well-known bound for the Mills' ratio for 
the normal distribution, obtain that

$$
\begin{aligned}
\int_{M}^{\infty} f(x)(x & \left.-M^{H}\right) \exp \left\{-\left(x-M^{H}\right)^{2} / 2\right\} d x=e^{a M^{H}} \int_{M-M_{H}}^{\infty} y e^{-y^{2} / 2+a y} d y \\
& =e^{a M^{H}+a^{2} / 2}\left[\int_{M-M_{H}}^{\infty}(y-a) e^{-(y-a)^{2} / 2} d y+a \int_{M-M_{H}}^{\infty} e^{-(y-a)^{2} / 2} d y\right] \\
& \leq e^{a M^{H}+a^{2} / 2}\left(1+\frac{a}{M-M^{H}-a}\right) e^{-\left(M-M^{H}-a\right)^{2} / 2} \\
& =e^{a M^{H}+a^{2} / 2}\left(1+\frac{a}{\left|2 \ln \Delta_{n}^{H}\right|^{1 / 2}}\right) \Delta_{n}^{H}
\end{aligned}
$$

Therefore

$$
\Delta_{n}^{H, f} \leq e^{a M^{H}+a^{2} / 2}\left(1+e^{a^{2} / 2+a\left|2 \ln \Delta_{n}^{H}\right|^{1 / 2}}+\frac{a}{\left|2 \ln \Delta_{n}^{H}\right|^{1 / 2}}\right) \Delta_{n}^{H} .
$$

Example 2. For the function $f(x)=x^{p}, p \geq 1$, one clearly has

$$
\Delta_{n}^{H, f} \leq p M^{p-1} \Delta_{n}^{H}+\int_{M}^{\infty} x^{p}\left(x-M^{H}\right) \exp \left\{-\left(x-M^{H}\right)^{2} / 2\right\} d x .
$$

Observe that $x^{p}=\left(x-M^{H}\right)^{p}\left(1+\frac{M^{H}}{x-M^{H}}\right)^{p} \leq\left(x-M^{H}\right)^{p}\left(\frac{M}{M-M^{H}}\right)^{p}$ for $x \geq M$, while, for any $A>0$,

$$
\int_{A}^{\infty} z^{p+1} e^{-z^{2} / 2} d z=-\int_{A}^{\infty} z^{p} d e^{-z^{2} / 2}=A^{p} e^{-A^{2} / 2}+p \int_{A}^{\infty} z^{p-1} e^{-z^{2} / 2} d z,
$$

where the last integral does not exceed $A^{-2} \int_{A}^{\infty} z^{p+1} e^{-z^{2} / 2} d z$, so that

$$
\int_{A}^{\infty} z^{p+1} e^{-z^{2} / 2} d z \leq \frac{A^{p} e^{-A^{2} / 2}}{1-p A^{-2}} \quad \text { for } A^{2}>p .
$$

Hence, choosing $A:=M-M^{H}=\left|2 \ln \Delta_{n}^{H}\right|^{1 / 2}$, we obtain that, for $\Delta_{n}^{H}<e^{-p / 2}$,

$$
\Delta_{n}^{H, f} \leq\left(M^{H}+\left|2 \ln \Delta_{n}^{H}\right|^{1 / 2}\right)^{p-1}\left(p+\frac{M^{H}+\left|2 \ln \Delta_{n}^{H}\right|^{1 / 2}}{1-p\left|2 \ln \Delta_{n}^{H}\right|^{-1}}\right) \Delta_{n}^{H} .
$$

Finally, in the next corollary we use Theorem 1 to improve the known upper bound $M^{H}<16.3 H^{-1 / 2}$ for the expected maximum $M^{H}$ from Theorem 2.1(ii) in [5].

Corollary 2. Assume that $H$ is such that $2^{2 / H}$ is integer. Then

$$
M^{H}<1.695 H^{-1 / 2} \text {. }
$$

Remark 5. If $2^{2 / H}$ is not integer then, in the above formula, one can use instead of $H$ the largest value $\widetilde{H}<H$ such that $2^{2 / \widetilde{H}}$ is integer, i.e. $\widetilde{H}=2 / \log _{2}\left\lceil 2^{2 / H}\right\rceil$. This is so since it follows from Sudakov-Fernique's inequality (see e.g. Proposition 1.1 and Section 4 in [5]) that the expected maximum $M^{H}$ is a non-increasing function of $H$. 
Remark 6. Our new upper bound for $M^{H}$ can be used to improve Shao's upper bound from $\left[12\right.$ for Pickands' constant $\mathcal{H}_{H}$, which is a basic constant in the extreme value theory of Gaussian processes and is of interest in a number of applied problems. That constant appears in the asymptotic representation for the tail probability of the maxima of stationary Gaussian processes in the following way (see e.g. [1] ).

Assume that $\left(X_{t}\right)_{t \geq 0}$ is a stationary Gaussian process with zero mean and unit variance of which the covariance function $r(v):=\mathbf{E} X_{t} X_{t+v}$, satisfies the following relation: for some $C>0$ and $H \in(0,1]$, one has $r(t)=1-C|t|^{2 H}+o\left(|t|^{2 H}\right)$ as $t \rightarrow 0$. Then, for each fixed $T>0$ such that $\sup _{\varepsilon \leq t \leq T} r(t)<1$ for all $\varepsilon>0$,

$$
\mathbf{P}\left(\sup _{0 \leq t \leq T} X_{t}>u\right)=C^{1 /(2 H)} \mathcal{H}_{H}(2 \pi)^{-1 / 2} e^{-u^{2} / 2} u^{1 / H-1}(T+o(1)), \quad u \rightarrow \infty .
$$

It was shown in [12] that, for $H \in(0,1 / 2]$,

$$
\mathcal{H}_{H} \leq\left(2^{1 / 2} e H M^{H}\right)^{1 / H}
$$

Using our Corollary 2, we obtain the following new upper bound for Pickands' constant:

$$
\mathcal{H}_{H}<(42.46 H)^{1 /(2 H)}, \quad H \in(0,1 / 2],
$$

which is superior to Shao's bound

$$
\mathcal{H}_{H} \leq\left\{1.54 H+4.82 H^{1 / 2}(4.4-H \ln (0.4+1.25 / H))^{1 / 2}\right\}^{1 / H}, \quad H \in(0,1 / 2]
$$

(see (1.5) in [12]; there the notation $a:=2 H$ is used). Fore example, the ratio of our bound to Shao's equals 0.344 when $H=0.45$ and is 0.046 when $H=0.15$.

\section{Proofs}

Proof of Theorem 11. First we will prove (3). Let $n_{k}:=n m^{k}, k \geq 0$, where we set $m:=\left\lfloor n^{\alpha}\right\rfloor \geq 2$. It follows from the continuity of $B^{H}$ and monotone convergence theorem that

$$
\Delta_{n}^{H}=\sum_{k=0}^{\infty}\left(M_{n_{k+1}}^{H}-M_{n_{k}}^{H}\right) .
$$

Although this step is common with the proof of Theorem 3.1 in [5], the rest of the argument uses a different idea. Namely, we apply Chatterjee's inequality ([6]; see also Theorem 2.2.5 in [1]) which, in its general formulation, states the following. For any $N$-dimensional Gaussian random vectors $X=\left(X_{1}, \ldots, X_{N}\right), Y=\left(Y_{1}, \ldots, Y_{N}\right)$ with common means: $\mathbf{E} X_{i}=\mathbf{E} Y_{i}$ for $1 \leq i \leq N$, one has

$$
\left|\mathbf{E} \max _{1 \leq i \leq N} X_{i}-\mathbf{E} \max _{1 \leq i \leq N} Y_{i}\right| \leq(\gamma \ln N)^{1 / 2}, \quad \gamma:=\max _{1 \leq i<j \leq N}\left|d_{i j}(X)-d_{i j}(Y)\right|,
$$

where, for a random vector $Z \in \mathbb{R}^{N}$, we set $d_{i j}(Z):=\mathbf{E}\left(Z_{i}-Z_{j}\right)^{2}, 1 \leq i, j \leq N$. 
To be able to apply inequality (8) to the terms in the sum on the right-hand side of (77), for each $k \geq 0$ we introduce auxiliary vectors $X^{k}, Y^{k} \in \mathbb{R}^{n_{k+1}}$ by letting

$$
X_{i}^{k}:=B_{i / n_{k+1}}^{H}, \quad Y_{i}^{k}:=B_{\lceil i / m\rceil / n_{k}}^{H}, \quad 1 \leq i \leq n_{k+1} .
$$

Note that $M_{n_{k+1}}^{H}=\mathbf{E} \max _{1 \leq i \leq n_{k+1}} X_{i}^{k}$ and $M_{n_{k}}^{H}=\mathbf{E} \max _{1 \leq i \leq n_{k+1}} Y_{i}^{k}$, so that now (8) is applicable. Next we will show that

$$
\gamma^{k}:=\max _{1 \leq i<j \leq n_{k+1}}\left|d_{i j}\left(X^{k}\right)-d_{i j}\left(Y^{k}\right)\right| \leq n_{k}^{-2 H}\left(1-m^{-1}\right)^{2 H} .
$$

Indeed, one can clearly write down the representations $i=a_{i} m+b_{i}, j=a_{j} m+b_{j}$ with integer $a_{j} \geq a_{i} \geq 0$ and $1 \leq b_{i}, b_{j} \leq m$, such that $b_{j}>b_{i}$ when $a_{i}=a_{j}$. Then it follows from (1) that

$$
d_{i j}\left(X^{k}\right)=\left(\frac{\left(a_{j}-a_{i}\right) m+b_{j}-b_{i}}{n_{k+1}}\right)^{2 H}, \quad d_{i j}\left(Y^{k}\right)=\left(\frac{\left(a_{j}-a_{i}\right) m}{n_{k+1}}\right)^{2 H} .
$$

Since for $2 H \leq 1$ the function $x \mapsto x^{2 H}, x \geq 0$, is concave, it is also sub-additive, so that $x^{2 H}-y^{2 H} \leq(x-y)^{2 H}$ for any $x \geq y \geq 0$. Setting $x:=d_{i j}\left(X^{k}\right) \vee d_{i j}\left(Y^{k}\right)$ and $y:=d_{i j}\left(X^{k}\right) \wedge d_{i j}\left(Y^{k}\right)$, this yields the desired bound

$$
\left|d_{i j}\left(X^{k}\right)-d_{i j}\left(Y^{k}\right)\right| \leq\left(\frac{\left|b_{i}-b_{j}\right|}{n_{k+1}}\right)^{2 H} \leq\left(\frac{m-1}{n_{k+1}}\right)^{2 H}=\frac{1}{n_{k}^{2 H}}\left(1-\frac{1}{m}\right)^{2 H} .
$$

Now it follows from (8) that

$$
\begin{aligned}
M_{n_{k+1}}^{H}-M_{n_{k}}^{H} & \equiv \mathbf{E} \max _{1 \leq i \leq n_{k+1}} X_{i}^{k}-\mathbf{E} \max _{1 \leq i \leq n_{k+1}} Y_{i}^{k} \\
& \leq\left(\gamma^{k} \ln n_{k+1}\right)^{1 / 2} \leq \frac{\left(1-m^{-1}\right)^{H}}{n^{H} m^{k H}}(\ln n+(k+1) \ln m)^{1 / 2} \\
& \leq \frac{(\ln n)^{1 / 2}}{n^{H}}\left(1-m^{-1}\right)^{H} \frac{(1+\alpha+\alpha k)^{1 / 2}}{m^{k H}}
\end{aligned}
$$

The last bound together with (7) leads to

$$
\Delta_{n}^{H} \leq \frac{(\ln n)^{1 / 2}}{n^{H}}\left(1-m^{-1}\right)^{H} \sum_{k=0}^{\infty} \frac{(1+\alpha+\alpha k)^{1 / 2}}{m^{k H}} .
$$

The sum of the series on the right hand side is exactly $\alpha^{1 / 2} \Phi\left(m^{-H},-\frac{1}{2}, 1+\alpha^{-1}\right)$, where $\Phi$ is the Lerch transcendent function. For our purposes, however, it will be convenient just to use the elementary bound $(1+\alpha+\alpha k)^{1 / 2} \leq(1+\alpha)^{1 / 2}(1+\alpha /(1+$ $\alpha))^{k / 2}$, to get

$$
\Delta_{n}^{H} \leq \frac{(\ln n)^{1 / 2}}{n^{H}} \cdot \frac{\left(1-m^{-1}\right)^{H}(1+\alpha)^{1 / 2}}{1-m^{-H}(1+\alpha /(1+\alpha))^{1 / 2}} .
$$

The right inequality in (3) is proved. To establish the left one, note that, on the one hand, it was shown in Theorem $2.1\left[5\right.$ ] that $M^{H} \geq L H^{-1 / 2}$ for all $H \in(0,1)$. 
On the other hand, it follows from Sudakov-Fernique's inequality (see e.g. Proposition 1.1 in [5]) that, for any fixed $n \geq 1$, the quantity $M_{n}^{H}$ is non-increasing in $H$, and it follows from Lemma 4.1 in [5] that

$$
M_{n}^{0}:=\lim _{H \rightarrow 0} M_{n}^{H}=2^{-1 / 2} \mathbf{E} \bar{\xi}_{n}, \quad \bar{\xi}_{n}:=\max _{1 \leq i \leq n} \xi_{i},
$$

where $\xi_{i}$ are i.i.d. $N(0,1)$-distributed random variables. Furthermore, the last expectation admits the following upper bound:

$$
\mathbf{E} \bar{\xi}_{n} \leq \sqrt{2 \ln n}, \quad n \geq 1
$$

Although that bound has been known for some time, we could not find a suitable literature reference or stable Internet link for it. So we decided to include a short proof thereof for completeness' sake. By Jensen's inequality, for any $s \in \mathbb{R}$,

$$
e^{s \mathbf{E} \bar{\xi}_{n}} \leq \mathbf{E} e^{s \bar{\xi}_{n}}=\mathbf{E} \max _{1 \leq i \leq n} e^{s \xi_{i}} \leq \mathbf{E} \sum_{1 \leq i \leq n} e^{s \xi_{i}}=\sum_{1 \leq i \leq n} \mathbf{E} e^{s \xi_{i}}=n e^{s^{2} / 2}
$$

so that $\mathbf{E} \bar{\xi}_{n} \leq s^{-1} \ln n+s / 2$. Minimizing in $s$ the expression on the right-hand side yields the desired bound (9).

From the above results, we obtain that

$$
\begin{aligned}
M^{H}-M_{n}^{H} & \geq M^{H}-M_{n}^{0} \geq L H^{-1 / 2}-(\ln n)^{1 / 2} \\
& =n^{-H}(\ln n)^{1 / 2} \cdot n^{H}\left(L(H \ln n)^{-1 / 2}-1\right),
\end{aligned}
$$

which completes the proof of Theorem 1,

Proof of Corollary [1. Since $f \geq 0$, for any $M>M^{H}$ we have

$$
\begin{aligned}
\Delta_{n}^{H, f} & \leq \mathbf{E}\left(f\left(\bar{B}_{1}^{H}\right)-f\left(\bar{B}_{n, n}^{H}\right) ; \bar{B}_{1}^{H} \leq M\right)+\mathbf{E}\left(f\left(\bar{B}_{1}^{H}\right) ; \bar{B}_{1}^{H}>M\right) \\
& \leq \omega_{\Delta_{n}^{H}, M}(f)+\int_{M}^{\infty} f(x) d F(x),
\end{aligned}
$$

where $F(x):=\mathbf{P}\left(\bar{B}_{1}^{H} \leq x\right)$. From the well-known Borell-TIS inequality for Gaussian processes (see, e.g., Theorem 2.1.1 in [1]) it follows that, for any $u>0$,

$$
\mathbf{P}\left(\bar{B}_{1}^{H}-M^{H}>u\right) \leq e^{-u^{2} / 2} .
$$

Therefore, for any $M>M^{H}$, integrating by parts, using the assumed property that $f(x) \exp \left\{-\left(x-M^{H}\right)^{2} / 2\right\} \rightarrow 0$ as $x \rightarrow \infty$, and then again integrating by parts, we can write

$$
\begin{aligned}
\int_{M}^{\infty} & f(x) d F(x)=f(M)(1-F(M))+\int_{M}^{\infty}(1-F(x)) d f(x) \\
& \leq f(M) \exp \left\{-\left(M-M^{H}\right)^{2} / 2\right\}+\int_{M}^{\infty} \exp \left\{-\left(x-M^{H}\right)^{2} / 2\right\} d f(x) \\
& =-\int_{M}^{\infty} f(x) d \exp \left\{-\left(x-M^{H}\right)^{2} / 2\right\} .
\end{aligned}
$$

Together with (10) this establishes the assertion of Corollary 1 . 
Proof of Corollary [2. Using Chatterjee's inequality (8) with the zero vector $Y$, we get for any $n \geq 1$ the bound $M_{n}^{H} \leq\left(\left(1-n^{-2 H}\right) \ln n\right)^{1 / 2}$, so that we obtain from Theorem 1 that

$$
\begin{aligned}
M^{H} & \leq \Delta_{n}^{H}+\left(\left(1-n^{-2 H}\right) \ln n\right)^{1 / 2} \\
& <\left[\frac{n^{-H}(1+\alpha)^{1 / 2}}{1-m^{-H}(1+\alpha /(1+\alpha))^{1 / 2}}+\left(1-n^{-2 H}\right)^{1 / 2}\right](\ln n)^{1 / 2} .
\end{aligned}
$$

Now choosing $n:=4^{1 / H}$ (which was assumed to be integer) and $\alpha:=2$, we get $m=n^{\alpha}=4^{2 / H}$ and

$$
M^{H}<H^{-1 / 2}\left[\frac{4^{-1} 3^{1 / 2}}{1-16^{-1}(5 / 3)^{1 / 2}}+\left(1-16^{-1}\right)^{1 / 2}\right](\ln 4)^{1 / 2}<1.695 H^{-1 / 2} .
$$

Acknowledgements. This research was supported by the ARC Discovery grant DP150102758. The work of M. Zhitlukhin was supported by the Russian Science Foundation project 14-21-00162.

\section{References}

[1] R. J. Adler and J. E. Taylor (2007) Random Fields and Geometry. Springer, New York

[2] V. F. Araman and P. W. Glynn (2012) Fractional Brownian motion with $H \leq$ $1 / 2$ as a limit of scheduled traffic. J. Appl. Probab. 49, 710-718

[3] C. Bayer, P. Friz and J. Gatheral (2016) Pricing under rough volatility. Quant. Finance, 16, 887-904

[4] F. Biagini, Y. Hu, B. Øksendal and T. Zhang. (2008) Stochastic Calculus for Fractional Brownian Motion and Applications. Springer, London

[5] K. Borovkov, Yu. Mishura, A. Novikov and M. Zhitlukhin . (2017) Bounds for expected maxima of Gaussian processes and their discrete approximations. Stochastics, 89, 21-37

[6] S. Chatterjee. (2005) An error bound in the Sudakov-Fernique inequality. arXiv:math/0510424

[7] N. Kordzakhia, Yu. Kutoyants, A. Novikov and T. Ling (2017) On a representation of fractional Brownian motion and the limit distributions of statistics arising in cusp statistical models. arXiv:math.st/1880353.

[8] Yu. Mishura (2008) Stochastic Calculus for Fractional Brownian Motion and Related Processes. Springer, Berlin-Heidelberg 
[9] I. Nourdin (2012) Selected Aspects of Fractional Brownian Motion. Springer, Milano

[10] A. Novikov, N. Kordzakhia and T. Ling (2014) On moments of Pitman estimators: the case of fractional Brownian motion. Theory Probab. Appl. 58, 601-614

[11] J. Pickands III. (1969) Asymptotic properties of the maximum in a stationary Gaussian process. Trans. Amer. Math. Soc. 145, 75-86

[12] Q.-M. Shao (1996) Bounds and estimators of a basic constant in extreme value theory of Gaussian processes. Statist. Sinica, 6, 245-257

[13] D. Siegmund (1979) Corrected diffusion approximations in certain random walk problems. Adv. Appl. Probab., 11, 701-710 